Clinical Article

\title{
Imaging Approach to Suspected Vascular Anomalies in Children
}

\author{
Eva Vič̌ič ${ }^{1}$, Damjana Ključevšek ${ }^{2}$ \\ ${ }^{1}$ General hospital "Dr. Franca Derganca" \\ Nova Gorica, ${ }^{2}$ University Children's \\ Hospital, University Medical Centre \\ Ljubljana
}

\author{
Correspondence: \\ evavicic@gmail.com \\ Tel.: + 38640279506 \\ Fax.: + 38515222993
}

Received: July 21, 2019

Accepted: August 20, 2019

Key Words: Vascular Anomalies Classification - Imaging - Ultrasound • Children.

\begin{abstract}
The purpose of this review is to become familiar with the terminology and typical presentation of vascular anomalies in children and to present the imaging approach to these anomalies. Vascular anomalies are the most common cause of soft-tissue masses in children. Many physicians are not familiar with the correct terminology for vascular anomalies which results in errors in diagnosis, prognosis, and treatment. The updated International Society for the Study of Vascular Anomalies classification, based on the original Hamburg classification, divides vascular anomalies into two types: vascular tumours (haemangiomas) and vascular malformations (venous, lymphatic, arteriovenous). Grey-scale and Doppler ultrasound (US) are the initial imaging modalities in suspected vascular anomalies. Grey-scale US is highly nonspecific. Therefore, a diagnostic algorithm based on grey-scale and Doppler signs is proposed. Spectral Doppler analysis reveals the type of vascular flow present within the lesion: high-flow is typical for infantile haemangiomas and arteriovenous malformations, low-flow for venous and noflow for lymphatic malformations. Magnetic resonance imaging with angiography is indicated only when US has not been diagnostic, when the lesion is too big or lies too deeply, and when information about the accurate anatomic location and possible infiltration of surrounding structures is necessary. Digital subtraction angiography is performed when interventional treatment is considered. Conclusion - The new terminology of vascular anomalies should be used in everyday clinical practice to avoid misunderstandings. Grey-scale and Doppler US are the most important imaging modalities for the accurate diagnosis and follow-up of vascular anomalies in children.
\end{abstract}

\section{Introduction}

Vascular anomalies are the most common cause of soft-tissue masses in children. MacFie et al. found that misdiagnosis of vascular anomalies is high: the initial diagnosis given to parents was inaccurate in $69 \%$ of patients and $53 \%$ of parents were given an incorrect account of what to expect with regard to the progression of the anomaly and the prospects for future treatment (1). Therefore, it is important to educate different profiles of medical doctors who work with children so they are familiar with the updated International Society for the Study of Vascular Anomalies (ISSVA) classification (2-4) based on the original Hamburg classification from 1988 (5). The latter divides vascular anomalies into two types: vascular tumours (haemangiomas), which are neoplastic lesions characterized by proliferation of endothelial cells, and vascular malformations, where an error in vascular morphogenesis occurs (5). 
The type of vascular malformation depends on the type of vessel affected. The most common are venous malformations (VM) (38\%), followed by lymphatic malformations (LM) (33\%), arteriovenous malformations (AVM) $(12 \%)$, combined malformations $(9 \%)$, and unclassified malformations (8\%) (6).

Prior to imaging investigation, it is necessary to obtain relevant clinical information and perform a clinical examination of the soft-tissue lump in order to narrow down the diagnostic possibilities. The history must contain information about the age of the child, the duration of the presence of the mass (was it present at birth?), its pattern of growth, changes in its appearance and the painfulness of the mass. It is also important to ask whether there are any associated systemic symptoms and whether any pre-existing conditions that may be associated with specific tumours are present, or whether there is a family history of similar lesions. During the clinical examination it is important to note the location of the mass, observe whether there are any overlying skin abnormalities, assess the consistency of the soft-tissue mass, its relation to surrounding tissues, and look for the presence of pulsations. Ultrasound (US) of a soft-tissue lump is the imaging modality of choice.

The purpose of this review is to become familiar with the new terminology and imaging approach in children with suspected vascular anomalies, and to be able to recognise typical vascular anomalies presenting in children based on grey-scale and Doppler imaging.

\section{Imaging Approach in Children with Suspected Vascular Anomalies}

In most cases, the diagnosis of a vascular anomaly is made on the basis of history, clinical examination, and grey-scale and Doppler US. These two techniques are the first imaging modalities of choice in suspected vascular anomalies. US examination is performed with a linear-array transducer of the highest frequency available $(14-22 \mathrm{MHz})$ for more superficial lesions, and a convex-array lowerfrequency transducer (5-9 MHz) for larger and deeper lesions.

If the history and clinical examination are typical for haemangioma, imaging is not required (2). Imaging is indicated only in clinically atypical haemangiomas, when the size, depth, or vascularity of the haemangioma has to be determined, and to evaluate the treatment effect. Grey-scale US provides information about the location of the lesion (confined to the subcutaneous tissue or infiltrating underlying muscles), its structure (solid, cystic), size, and borders (well or illdefined). Because grey-scale US can be highly nonspecific, spectral and colour Doppler US must also be performed. Information about intralesional flow, vessel location (central, peripheral, or both), and the degree of vascularisation (vessel density - the number of colour flow signals per square centimetre within an area of greatest intralesional vascularity) is obtained by colour Doppler US. On spectral Doppler US, the peak velocity of arterial and venous flow, resistive index (RI), the pulsatility of venous flow, and arteriovenous shunts are determined. Doppler US helps classify vascular anomalies into high-flow (haemangiomas, AVM, arteriovenous fistulas) and low- or no-flow vascular lesions (capillary malformations, VM, LM) based on the type of flow present within the lesion (Fig. 1) (7).

Magnetic resonance (MR) imaging is the diagnostic modality of choice when US has not been diagnostic, when the lesion is too big or lies too deeply, and when the information about the accurate anatomic location and possible infiltration of surrounding structures (muscle, tendons, nerves, and even bones) is necessary. A combination of T1-weighted and T2-weighted images with fat suppression added to a T2-weighed pulse sequence and sometimes a gradient-echo se- 


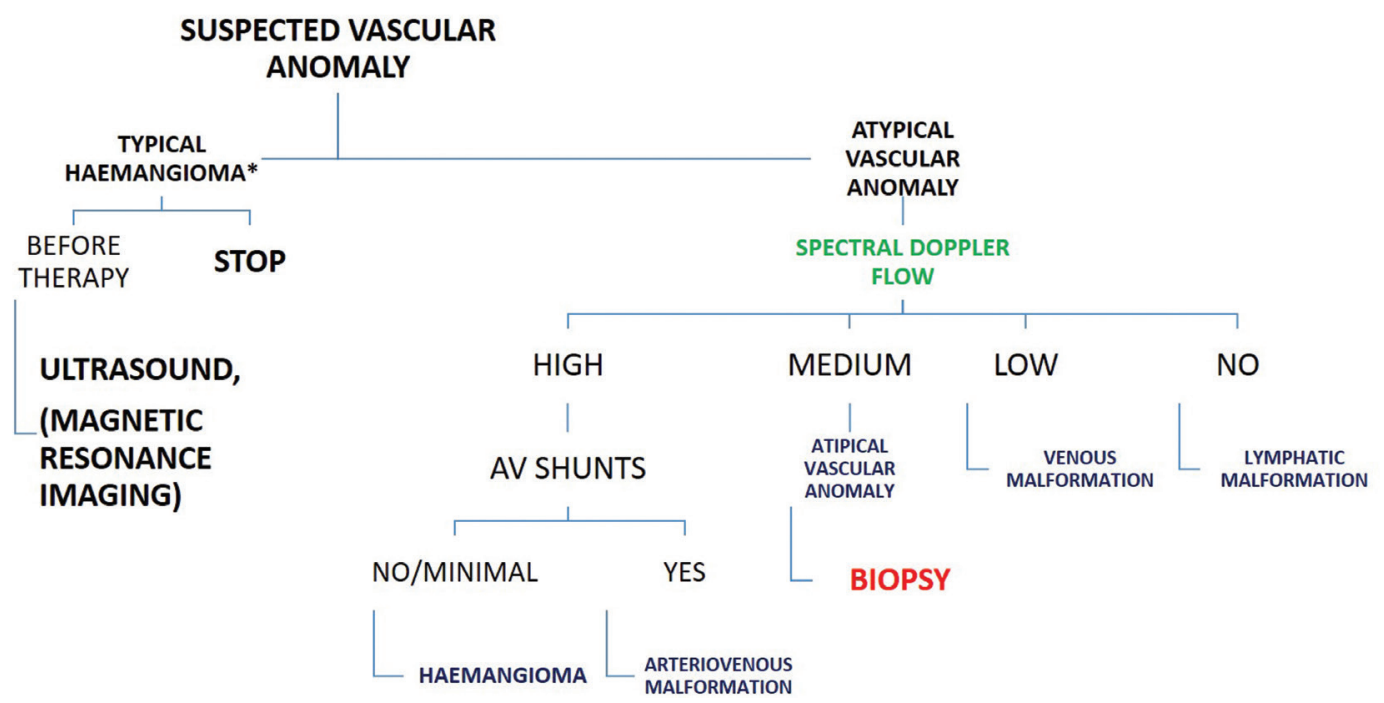

Fig. 1. Diagnostic Algorithm of Vascular Anomalies, Adapted from Hemangioma vs Vascular Malformation by Dubois and Garel (7). "The Diagnosis of Typical Haemangioma Is Based on History and Clinical Examination.

quence (for the detection of hemosiderin and high-flow vessels) are the basis of MR imaging of vascular anomalies. To differentiate between venous and lymphatic malformations, the administration of intravenous gadolinium chelates is frequently used. When AVM is suspected, MR angiography is performed in order to detect the feeding artery and venous outflow. MR and US are complementary methods. The advantage of MR is the high level of intrinsic contrast, and its capacity for multiplanar imaging. However, MR imaging does not offer the same capability of real time imaging that US does. Digital subtraction angiography (DSA), which allows optimal visualisation of vessel anatomy, is usually performed when interventional treatment, such as embolisation, is considered. In challenging cases, particularly when a well-vascularized soft-tissue mass with moderate flow is detected, a biopsy should be performed to exclude a soft tissue tumour (Fig. 2). However, some vascular lesions still remain unclassified despite all imaging and histology.

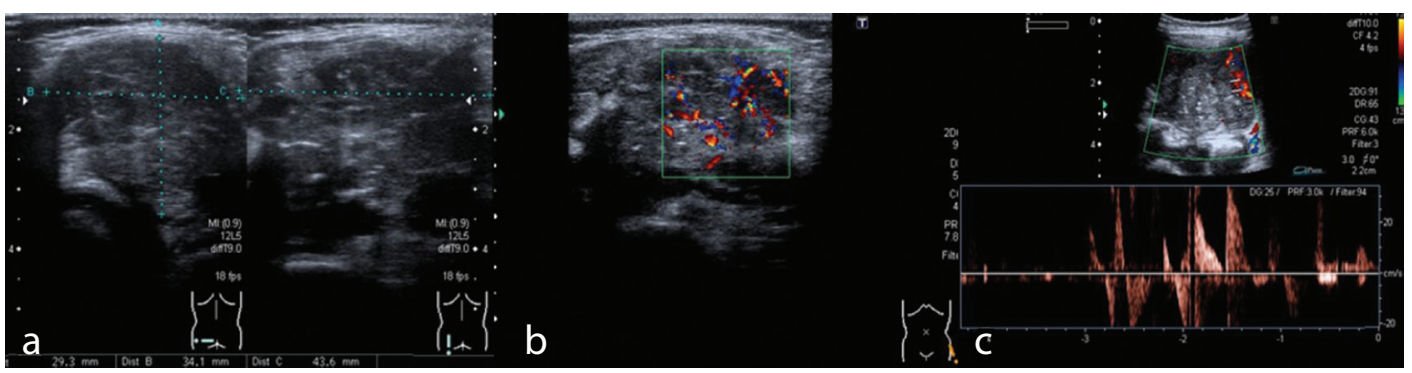

Fig. 2. Embryonal Rhabdomyosarcoma in a 2.5-Month-Old-Boy with a Palpable, Non-Tender Soft Tissue Mass in the Gluteal Region. A: Well-Defined, Slightly Hypoechoic Heterogeneous Mass Is Seen on Gray-Scale Ultrasound. B: Color Doppler Reveals High Vascular Density of the Mass. C: Spectral Doppler Analysis Shows Chaotic Waveform Patterns with High Velocities, Arteriovenous Shunts and an Irregular Curve. 


\section{Clinical and Us Imaging Characteristics of Vascular Anomalies}

\section{Vascular Tumours}

Vascular tumours are high-flow vascular, neoplastic lesions, characterized by proliferation of endothelial cells that have a high turnover with great potential for growth.

Infantile Haemangioma is the most common vascular tumour in infants, occurring in up to $10 \%$ of all infants by 1 year of age. It is not present at birth, but a flat, pink, prodromal lesion might be seen, which then progresses into a bright red mass a couple of weeks after birth. Infantile haemangioma exhibits a characteristic two phase natural history. Rapid growth of haemangiomas occurs during the first months (proliferation phase) (Fig. 3), then slows down and stabilizes. At approximately 1 to 2 years of age the involution phase begins, which is much slower than the proliferation phase (Fig. 4). $90 \%$ of infantile haemangiomas reach maximal involution by the age of 9 years (8). They are usually solitary and most frequently found in the face and neck area (60\%). Imaging is usually required in only $10 \%$ of cases, when we encounter atypical or deep lesions. The most common complications of haemangiomas are cutaneous bleeding and ulceration. Infantile haemangiomas may be associated with various congenital syndromes (PHACE or LUMBAR), which warrants further investigation, so it is important to note any underlying developmental disorder the child might also have (Table 1).

On US we see a well-defined hypoechoic solid lesion, although hyperechoic and heterogeneous appearances have also been described (9-11). Intralesional vessels are rarely seen on grey-scale US. In the proliferative phase, there is high vascular density (more than 5 vessels per square centimetre in the area of greatest vascularisation). Both arterial and venous flow is usually present on Doppler imaging, but arteriovenous shunting is rare (Fig. 3) (7).

In the involutive phase, there is a decrease in size and vascular density and an increase in echogenicity due to fibro-fatty replacement (Fig. 4) (12). Possible complications are infection, cutaneous bleeding, and ulceration, which occur in 5 to $15 \%$ of focal lesions (1315).

Congenital Haemangiomas are already fully grown at birth, because their prolifera-

\section{Table 1. Explanation of the Syndrome Acronyms}

\begin{tabular}{ll}
\hline Syndrome & Explanation of the acronym \\
\hline PHACE & Posterior fossa brain malformations, Haemangiomas of the face, neck or scalp, \\
& Arterial anomalies, \\
& Coarctation of the aorta or Cardiac defects, \\
& Eye abnormalities \\
\hline LUMBAR & Lower body infantile haemangiomas, \\
& Urogenital anomalies, Ulceration, \\
& Myelopathy, \\
& Bony deformities, \\
& Anorectal malformations, Arterial anomalies, \\
& Renal anomalies \\
\hline CLOVES & Congenital lipomatous overgrowth, \\
& Vascular malformations, \\
& Epidermal naevi, \\
& Skeletal anomalies \\
\hline FAVA & Fibro-adipose vascular anomaly \\
\hline
\end{tabular}




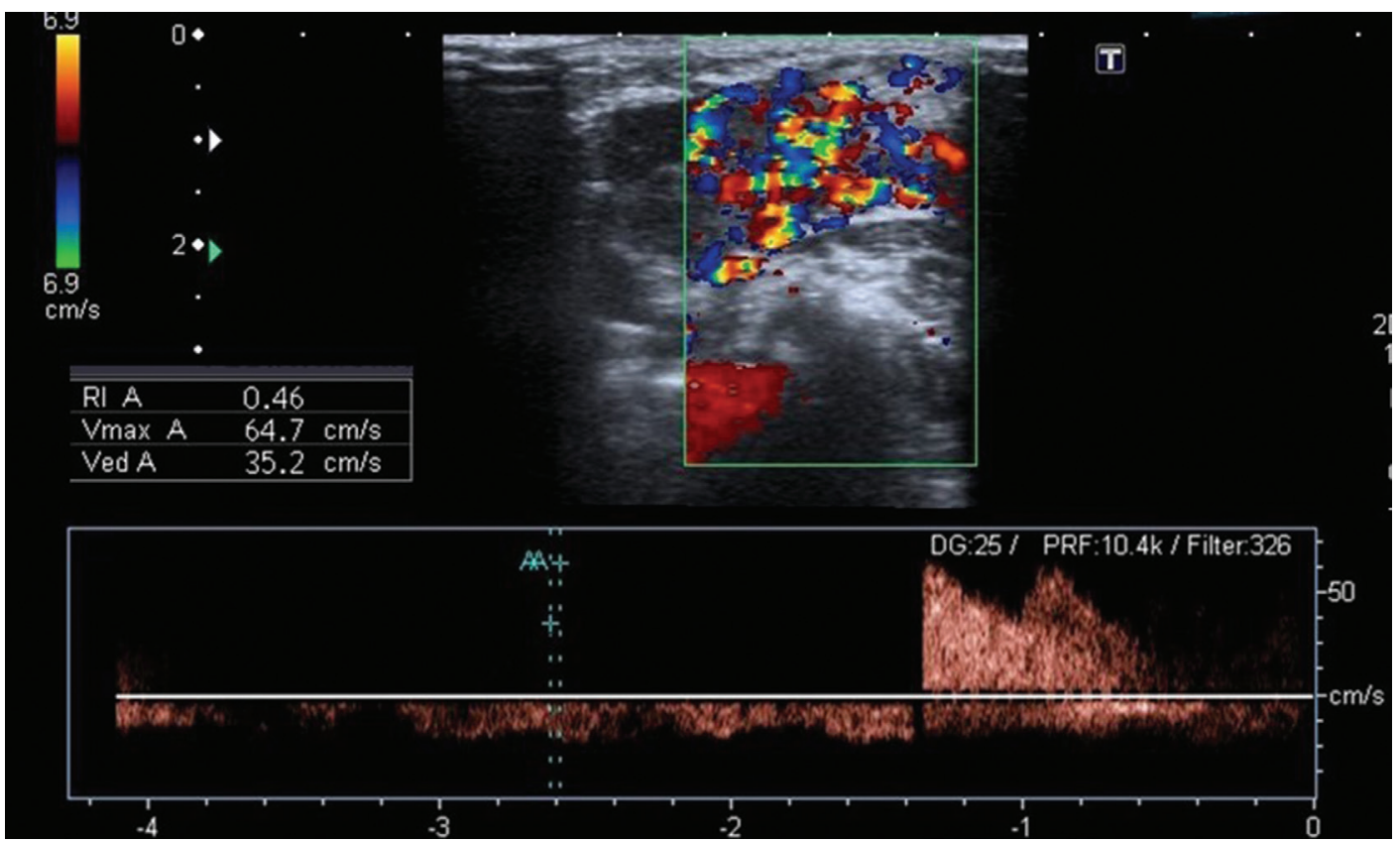

Fig. 3. Infantile Haemangioma in the Proliferative Phase in a 4-Month-Old Boy. Colour Doppler Imaging Reveals the High Vascular Density of the Mass.

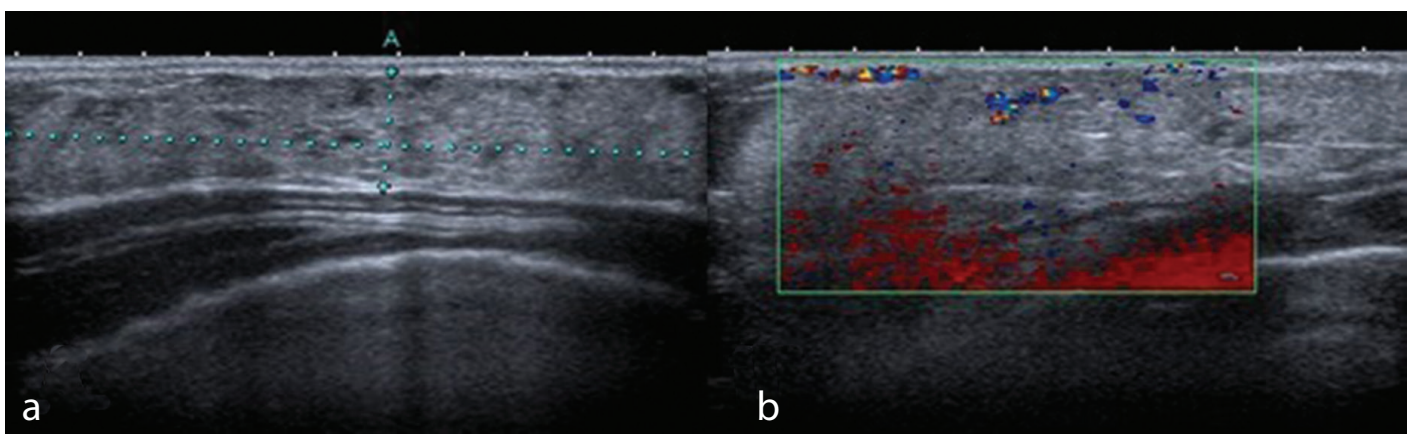

Fig. 4. Infantile Haemangioma in the Involutive Phase in an 1-Year-Old Girl. A: A Hyperechoic Mass with Tiny Hypoechoic Areas Is Seen on Grey-Scale Ultrasound. B: Colour Doppler Shows the Low Vascular Density of the Mass.

tive phase started in utero. Three subtypes exist: rapidly involuting congenital haemangioma (RICH), non-involuting congenital haemangioma $(\mathrm{NICH})(16,17)$ and partially involuting congenital haemangioma (PICH) (18). As the name suggests NICH does not exhibit involution, which occurs with RICH by 14 months of age. In contrast to haemangiomas of infancy, they are more often heterogeneous on US and calcifications are more frequently seen. The heterogeneous echo- genicity is mostly due to the intralesional vessels (usually veins) that are visible on greyscale US. The presence of venous clots, which are never found in infantile haemangiomas, has also been reported (19).

Pyogenic Granuloma is a lobular capillary haemangioma, which presents as a quickly growing, bleeding vascular skin lesion in children older than 6 months of age on the head, neck, or upper extremities. Most diagnoses are made clinically. Well-defined, 
small, hypoechoic lesions with high vascular density can be seen on US (20).

Kaposiform Haemangioendotheliomas usually present in the first year of life and are sometimes congenital. Their site of predilection is an extremity. Clinically they present with differing cutaneous appearance and discoloration. They can be tender, locally aggressive and can even involve adjacent bones. An associated hypertrichosis, if present, can be suggestive of the diagnosis. They may be associated with the Kasabach-Merritt's phenomenon, which is a systemic complication characterized by thrombocytopenia, microangiopathic haemolytic anaemia and secondary consumption of coagulation factors $(21,22)$. Its presence is indicative of either Kaposiform haemangioendothelioma or tufted angioma, which is a similar tumour. On US, Kaposiform haemangioendothelioma has variable echogenicity with poorly defined margins, and it is often difficult to detect and accurately evaluate the infiltration of surrounding tissues. MR imaging is necessary in most cases. It may contain calcifications. It may be focal or diffuse and may involve all soft-tissue planes, including underlying bone. Doppler analysis reveals a moderate degree of vascularisation. However, there are reports of tumours having high vascular density and vessels, visible on grey-scale US $(23,24)$.

Tufted Angiomas are similar to Kaposiform haemangioendotheliomas, only more superficial and thinner, not exceeding $1 \mathrm{~cm}$ in thickness, and are surrounded by hyperechoic subcutaneous fat (25).

Red Flags for a Possibly Malignant Lesion, such as sarcoma or haemangioendothelioma, include a painful mass in an older child, rapid growth of the lesion after 12 months of age (26), a history of concomitant lung, bone or liver lesions, and lymphadenopathy adjacent to the lesion. On imaging there might be soft-tissue or bone destruction and calcifications, haemorrhage or necrosis in a non-congenital lesion. These findings warrant further diagnostic imaging (MR), possibly coupled with biopsy.

\section{Vascular Malformations}

Vascular malformations are abnormal channels lined by normal endothelium because an error in vascular morphogenesis has occurred. The type of vascular malformation is dependent on the type of vascular endothelium. They are always present at birth, although many are not detected until later due to their slow growth (usually they grow with the child). They have a low turnover. More rapid growth may be caused by trauma, clotting and hormonal influence during puberty or pregnancy. Vascular malformations do not involute.

Venous Malformations are low-flow lesions composed of ectatic venous channels, and are the most common vascular malformation with a prevalence of $1 \%$ in the general population. The common misnomer for VM is cavernous haemangioma, which should not be used as they are non-neoplastic lesions. When VMs lie superficially, they appear as a soft-tissue mass with a bluish discoloration of the overlying skin, and are soft and compressible on palpation. Deeper lesions often lack cutaneous changes and are thus more difficult to diagnose. Their size is gravity-dependent and also increases with exertion or crying. They can be episodically tender, when a thrombosis is present, which is more common during puberty or pregnancy. Most commonly they are found in the head and neck region (40\%) and extremities (40\%). On US they appear as relatively well-defined, heterogeneous and mostly hypoechoic sponge-like masses, although isoechoic and hyperechoic appearances have also been described (Fig. 5A) (27). Demonstration of phlebolits, which are the foci of calcification, is almost pathognomonic for a VM, because 


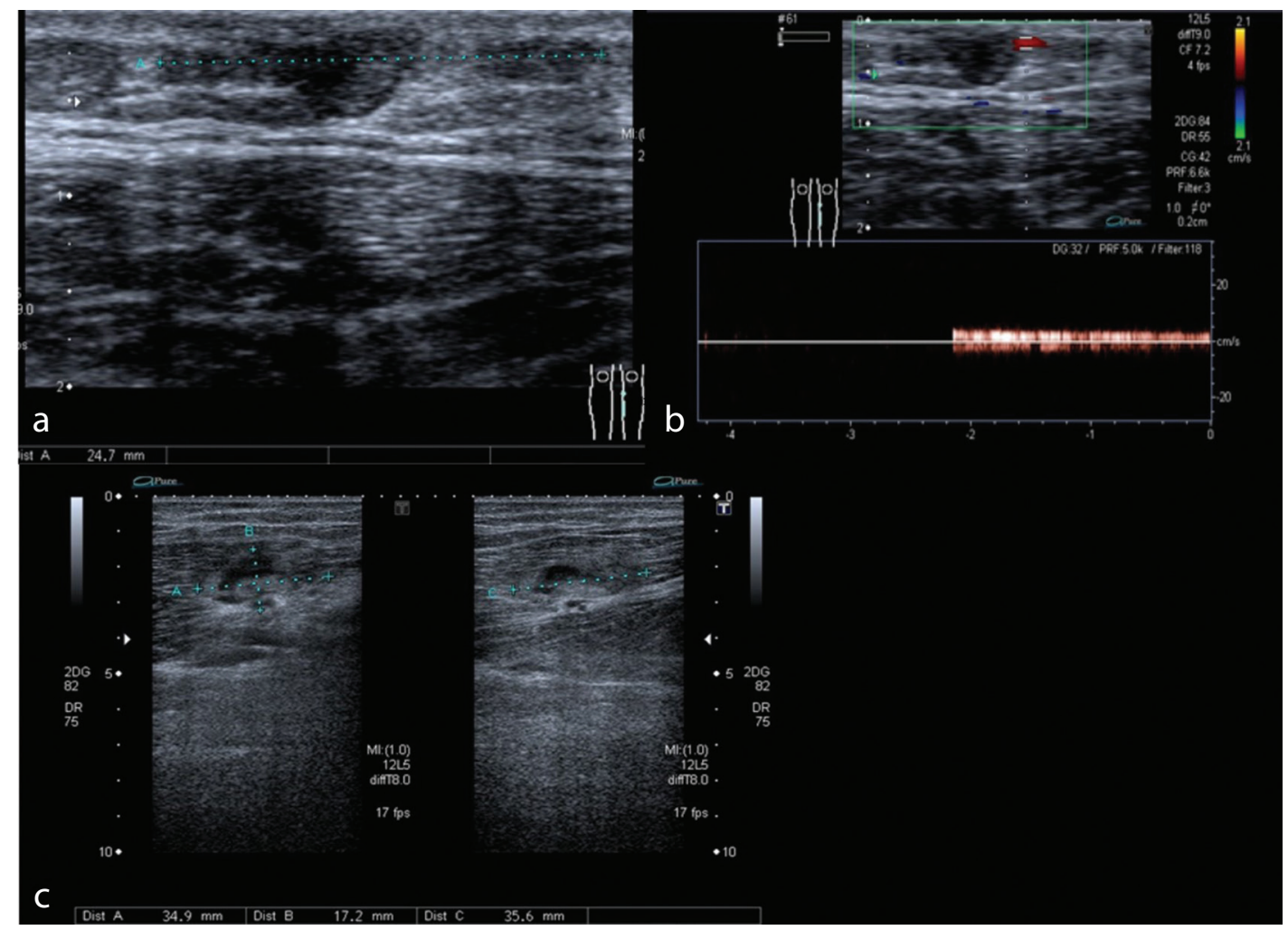

Fig. 5. Venous Malformation in A 14-Year-Old Boy with a Lesion in the Subcutaneous Tissue of The Medial Part of the Shank. A: An Irregular, Heterogeneous and Mostly Hypoechoic Mass Is Seen on Gray-Scale Ultrasound. B: Color and Spectral Doppler Analysis Shows Low Vascular Density within the Lesion with Low-Level Venous Flow. C: Gray-Scale Ultrasound Shows the Presence of an Intralesional Thrombus in a Venous Malformation.

they rarely occur in other vascular anomalies. Phlebolits, however, are rarely seen in children $(27,28)$. An intralesional thrombus is seen as a hyperechoic mass within the vein (Fig. 5C). Doppler interrogation shows low vascular density with low-level venous flow (Fig. 5B). It is not uncommon for no flow to be detected at all due to the very slow flow that is not detectable with conventional equipment. In that case, applying compression or performing the Valsalva manoeuvre might help enhance the detection of flow (19). Sometimes arterial flow can be detected within the lesion, which represents either the presence of a capillary malformation (combined vascular malformation) or normal arteries coursing through the lesion $(26,29)$.
Lymphatic Malformations are low-flow lesions, which can be microcystic, macrocystic or combined. Two misnomers for lymphatic malformations have previously been used in the literature: lymphangiomas and cystic hygromas. These terms should not be used as they imply that lymphatic malformations are neoplastic lesions, which is not the case. Although they may appear anywhere on the body, they tend to be slightly more common in the head and neck region. Microcystic LM consists of multiple microscopic cavities. Clinically they appear as grouped, clear or sometimes haemorrhagic vesicles, often with a deep component. On US they tend to be echogenic masses, measuring less than $1 \mathrm{~cm}$ (Fig. 6B, 6C). Sometimes they are so small that the cystic spaces are not seen, but 


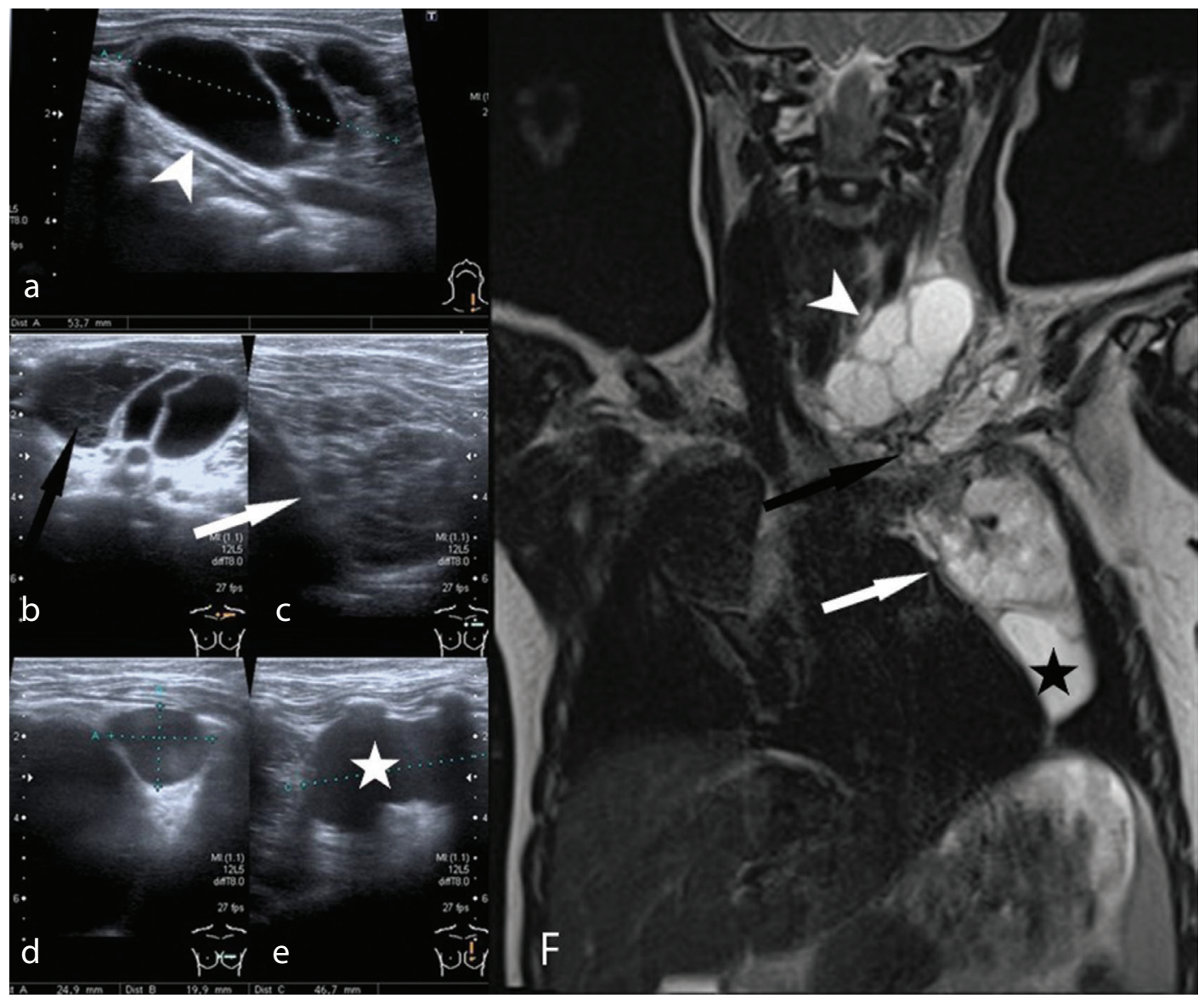

Fig. 6. A: Combined Macrocystic and Microcystic Lymphatic Malformation (LM) in the Neck and Thorax Region in a 7-Month-Old Girl. A-E: Multiple Anechoic Cystic Spaces Separated by Thin Septa Are Seen on GreyScale Ultrasound (US). A, D and E: Show the Macrocystic Component (White Arrowhead, White Star), and B and C: Show The Microcystic Component (Black Arrow, White Arrow) of the LM. F: Magnetic Resonance Imaging, Coronal T2-Weighted TSE Sequence. the White Arrowhead and Black and White Arrows Represent the Corresponding Cystic Spaces Seen on the US Image (A, B, C). The Black Star Represents the Cystic Space, Designated with a White Star on US Image (E).

the multiple interfaces caused by tiny cysts result in an ill-defined area of thickening and increased echogenicity. Macrocystic lesions display cystic spaces, with or without fluidfluid levels (Fig. 6A, 6D, 6E). Clinically they present as soft, smooth, translucent masses without abnormality or with bluish discoloration of the skin. On US they appear as large, septated anechoic cavities (Fig. 6). In most cases there are no vascular signals in the septa, although arterial and /or venous flow may be detected. However, no flow is demonstrated within the cystic spaces.

A large LM located in the neck that extends into the thoracic cavity requires MR imaging to evaluate the full size of the malformation and its relationship with the surrounding tissues (Fig. 6F). When a complication occurs, such as an infection or haemorrhage, they may contain echoes or fluiddebris levels due to the presence of proteinaceous material (Fig. 7). Compression with the transducer may deform the cystic spaces, 


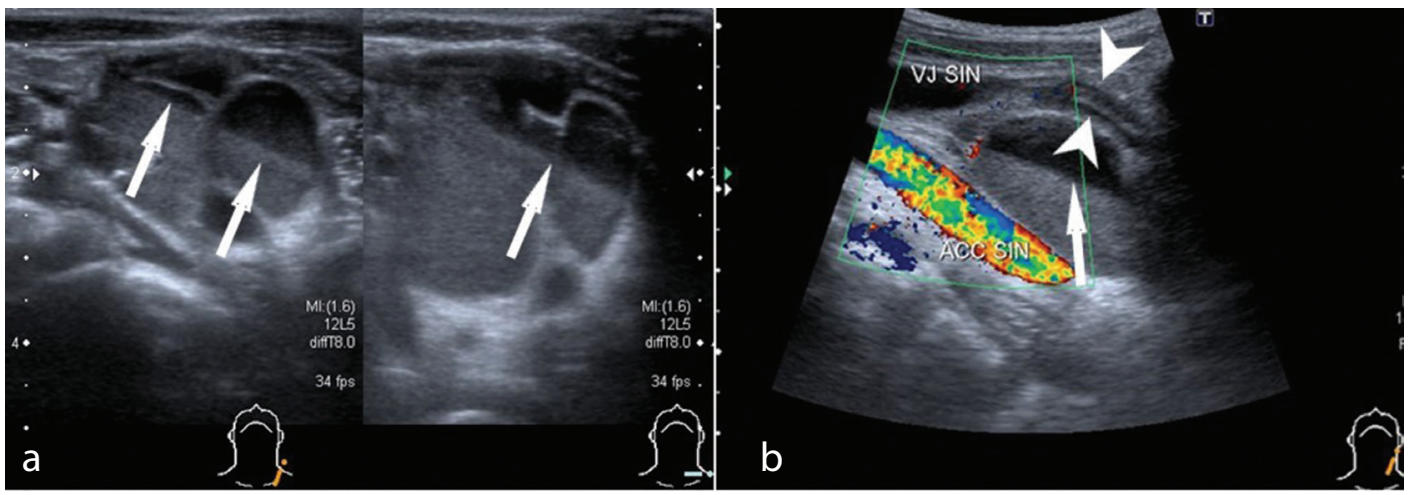

Fig. 7. A Macrocystic Lymphatic Malformation (LM) on the Neck of an 8-Year-Old Boy. A: Haemorrhage into the Macrocystic LM With Fluid-Fluid Levels (Arrows) Is Seen on Grey-Scale Ultrasound. B: The Compressed Left Jugular Vein (Arrowheads) Due To the Pressure of an Enlarged LM Is Seen on Colour Doppler Ultrasound.

but they do not collapse, which differentiates them from venous malformations (30).

Arteriovenous Malformations are highflow lesions with direct shunting between arteries and veins, without intervening arterioles or a capillary bed (31). Most are asymptomatic during childhood (Schobinger classi- fication stage I - dormancy) (32). In puberty or pregnancy, they begin to grow (stage II - expansion), which is thought to be caused by hormonal influences. Skin changes tend to occur, the local temperature increases, and thrill or pulsations might be present. Progression to stages III and IV is rare and usually

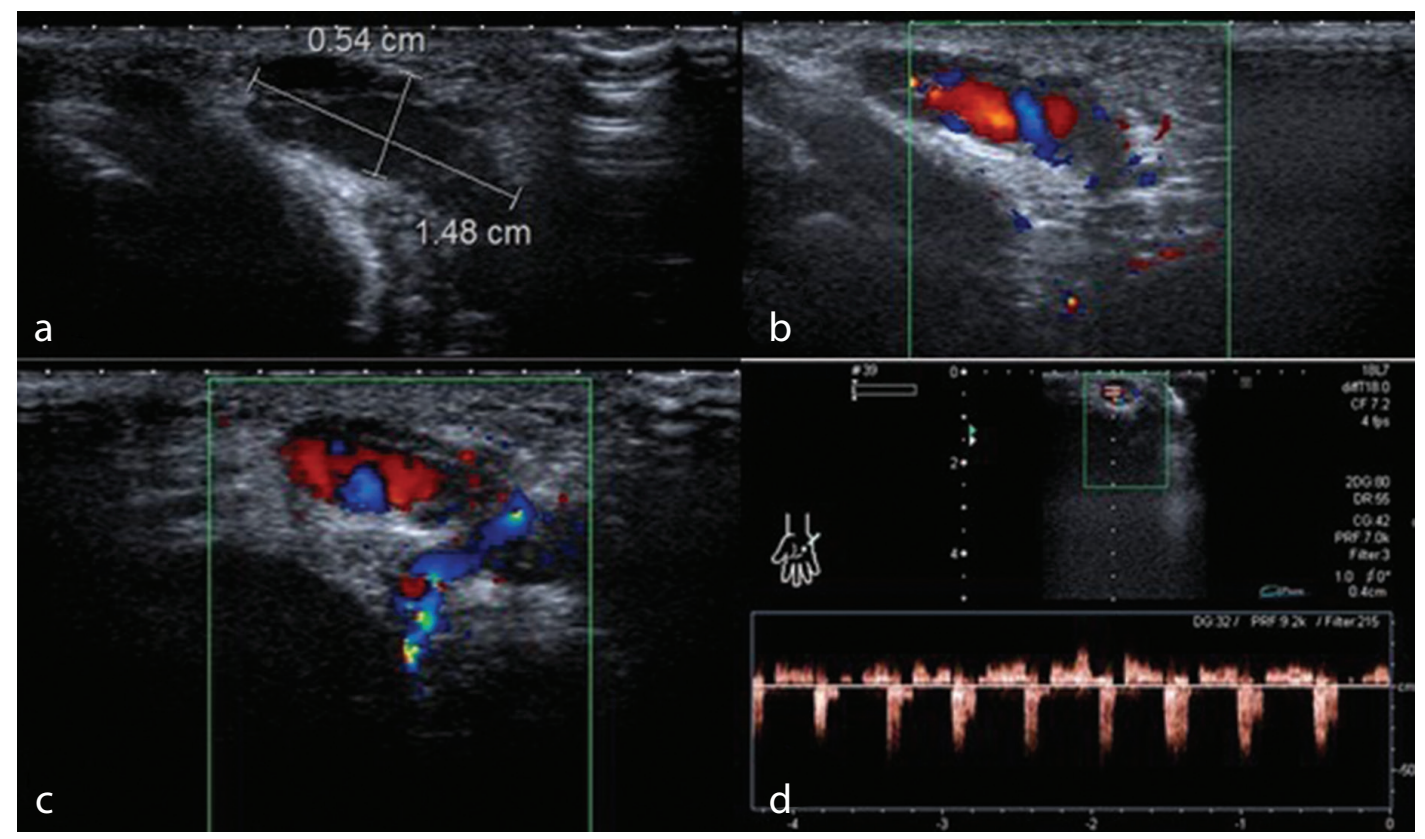

Fig. 8. Arteriovenous Malformation in a 9-Month-Old Girl Who Presented with a Compressible, Pulsatile Mass on the Palm. A: Hypoechoic Tortuous Vessels With No Discrete Solid Mass Are Seen On Grey-Scale Ultrasound. B, C: Colour Doppler Shows the High Vascular Density of the Mass. D: Spectral Doppler Analysis Reveals High-Flow Arterial Signals (Curve Below the Central Line) with Venous Flow and Shunt Signals (Above the Line). 
seen only in untreated cases. In stage III (destruction), skin necrosis, ulceration or haemorrhage and lytic bone lesions may appear. Stage IV begins when congestive heart failure develops in a stage III patient. Although an AVM is present at birth, it becomes clinically apparent usually after trauma or hormonal disturbances when rapid growth begins (4). In contrast to haemangioma of infancy, there is usually an absence of a solid mass on greyscale US. On Doppler US they exhibit high vascular density, high vascular arterial flow, pulsatile venous flow, signs of arteriovenous shunting, and low resistive index $(\mathrm{RI}<0.5)$ (Fig. 8).

Capillary Malformations usually do not require additional imaging, because of the obvious clinical diagnosis. A port wine stain, or nevus flameus, is the most common in the category and occurs in approximately $0.3 \%$ of newborns (8). It presents as a red or pink macule, usually in the head and neck region. Most commonly they are only of cosmetic concern, but may be associated with Sturge-Weber, Klippel-Trénaunay or Proteus syndrome. They are often not apparent on imaging. Sometimes a focal thickening of the skin and subcutaneous tissue is seen. With a specialized 20-24 MHz transducer, capillary malformations may sometimes appear as superficial hypoechoic areas with a mean depth of $1 \mathrm{~mm}$ (4). On colour Doppler US a slightly increased vascular density compared to the adjacent dermis might be seen.

Various Complex Combined Vascular Anomalies, such as lympho-venous malformations, may be diagnosed in almost $10 \%$ of cases (6). Some vascular anomalies cannot be classified despite all imaging. Vascular Malformation Syndromes usually require MR imaging due to associated changes in the adjacent soft tissues and skeleton, including limb overgrowth, and the extensive involvement of the extremities, sometimes with extension into the trunk. Some vascular anom- alies cannot be classified despite all imaging. In this case a biopsy of the vascular anomaly can give more information. Of the syndromes affecting the extremities, some include highflow lesions: Parkes-Weber, Bannayan-RileyRuvalcaba and Cowden syndromes, rarely also CLOVES syndrome (33-35). The others (FAVA, CLOVES, Klippel-Trénaunay, Proteus and Maffucci syndromes) include lowflow lesions (36-38) (Table 1).

\section{Conclusion}

A multidisciplinary approach regarding the diagnosis and treatment of vascular lesions in children is mandatory. Physicians who deal with children should be familiar with the terminology of vascular lesions to avoid confusion and misunderstandings. Grey-scale and Doppler US are the first imaging modalities in children with a soft-tissue mass, and in suspected vascular anomalies in children. When the diagnosis cannot be reliably made on US, MR imaging is the next modality of choice. In challenging cases and when the MR investigation is inconclusive, a biopsy is the next diagnostic tool.

Conflict of Interest: The authors declare that they have no conflict of interest.

\section{References}

1. MacFie CC, Jeffery SL. Diagnosis of vascular skin lesions in children: an audit and review. Pediatr Dermatol. 2008;25(1):7-12.

2. Wassef M, Blei F, Adams D, Alomari A, Baselga E, Berenstein A et al. Vascular Anomalies Classification: Recommendations From the International Society for the Study of Vascular Anomalies. Pediatrics. 2015;136(1):e203-e14.

3. Johnson CM, Navarro OM. Clinical and sonographic features of pediatric soft-tissue vascular anomalies part 1: classification, sonographic approach and vascular tumors. Pediatr Radiol. 2017;47(9):1184-95. 
4. Johnson CM, Navarro OM. Clinical and sonographic features of pediatric soft-tissue vascular anomalies part 2: vascular malformations. Pediatr Radiol. 2017;47(9):1196-1208.

5. Belov S. Anatomopathological classification of congenital vascular defects. Semin Vasc Surg. 1993;6(4):219-24.

6. Lee BB. Changing Concept on Vascular Malformation: No Longer Enigma. Ann Vasc Dis. 2008;1(1):11-9.

7. Dubois J, Garel L. Imaging and therapeutic approach of hemangiomas and vascular malformations in the pediatric age group. Pediatr Radiol. 1999;29:879-93.

8. Frieden I, Enjolras O, Esterly N. Vascular birthmarks and other abnormalities of blood vessels and lymphatics. In: Schachner LA, Hansen RC, editors. Pediatric Dermatology. 3rd ed. New York: Mosby; 2003. p. 833-62.

9. Paltiel HJ, Burrows PE, Kozakewich HP, Zurakowski D, Mulliken JB. Soft-tissue vascular anomalies: utility of US for diagnosis. Radiology. 2000;214:747-54.

10. Dubois J, Patriquin HB, Garel L, Powell J, Filiatrault D, David $M$ et al. Soft-tissue hemangiomas in infants and children: diagnosis using Doppler sonography. AJR Am J Roentgenol. 1998;171:247-52.

11. Gorincour G, Kokta V, Rypens F, Garel L, Powell J, Dubois J. Imaging characteristics of two subtypes of congenital hemangiomas: rapidly involuting congenital hemangiomas and non-involuting congenital hemangiomas. Pediatr Radiol. 2005;35:1178-85.

12. Sheybani EF, Eutsler EP, Navarro OM, Fat-containing softtissue masses in children. Pediatr Radiol. 2016;46:1760-73.

13. Abernethy LJ. Classification and imaging of vascular malformations in children. Eur Radiol. 2013;13:2483-97.

14. Gampper TJ, Morgan RF. Vascular anomalies: Hemangiomas. Plast Reconstr Surg. 2002;110(2):572-85.

15. Waner M, North PE, Scherer K, Frieden IJ, Waner A, Mihm MC Jr. The non-random distribution of nonfacial hemangiomas. Arch Dermatol. 2003;139:869-75.

16. Boon LM, Enjolras O, Mulliken JB. Congenital hemangioma: evidence of accelerated involution. J Pediatr. 1996;128:329-35.
17. Enjolras O, Mulliken JB, Boon LM, Wassef M, Kozakewich HP, Burrows PE. Noninvoluting congenital hemangioma: a rare cutaneous vascular anomaly. Plast Reconstr Surg. 2011;107:1647-54.

18. Mulliken JB, Enjolras O. Congenital hemangiomas and infantile hemangioma: missing links. J Am Acad Dermatol. 2004;50:875-82.

19. Dubois J, Alison M. Vascular anomalies: what a radiologist needs to know. Pediatr Radiol. 2010;40(6):895-905.

20. Cantisani V, Del Vecchio A, Fioravanti E, Romeo U, D’Ambrosio F. Color-Doppler US features of a pyogenic granuloma of the upper dorsum tongue. J Ultrasound. 2016;19:67-70.

21. Colmenero I, Hoeger PH. Vascular tumours in infants. Part II: vascular tumours of intermediate dignity and malignant tumours. Br J Dermatol. 2014;171:474-84.

22. Kasabach H, Merritt K. Capillary hemangioma with extensive purpura: report of a case. Am J Dis Child. 1940;59:1063-70.

23. Johnson C, Navarro O. Clinical and sonographic features of pediatric soft-tissue vascular anomalies part 1: classification, sonographic approach and vascular tumors. Pediatr Radiol. 2017;47(9):118495.

24. Kollipara R, Dinneen L, Rentas KE, Saettele MR, Patel SA, Rivard DC et al. Current classification and terminology of pediatric vascular anomalies. AJR. 2013;201:1124-35.

25. Dubois J, Garel L, David M, Powell J. Vascular softtissue tumors in infancy: distinguishing features on Doppler sonography. AJR. 2002;178:1541-45.

26. Olivieri B, White C, Restrepo R, McKeon B, Karakas S, Lee E. Low-Flow Vascular Malformation Pitfalls: From Clinical Examination to Practical Imaging Evaluation-Part 2, Venous Malformation Mimickers. AJR. 2016;206(5):952-62.

27. Trop I, Dubois J, Guibaud L, Grignon A, Patriquin $\mathrm{H}$, McCuaig $\mathrm{C}$ et al. Soft-Tissue Venous Malformations in Pediatric and Young Adult Patients: Diagnosis with Doppler US. Radiology. 1999;212(3):841-5.

28. Paltiel H, Burrows P, Kozakewich H, Zurakowski D, Mulliken J. Soft-Tissue Vascular Anomalies: Utility of US for Diagnosis. Radiology. 2000;214(3):747-54.

29. Restrepo R. Multimodality imaging of vascular anomalies. Pediatr Radiol. 2013;43(S1):141-54. 
30. Morrow MS, Oliveira AM. Imaging of lumps and bumps in pediatric patients: an algorithm for appropriate imaging and pictorial review. Semin Ultrasound CT MR. 2014;35:415-29.

31. Uller W, Alomari AI, Richter GT. Arteriovenous malformations. Semin Pediatr Surg. 2014;23:2037.

32. Navarro OM. Magnetic resonance imaging of pediatric soft-tissue vascular anomalies. Pediatr Radiol. 2016;46(6):891-901.

33. Uller W, Fishman S, Alomari A. Overgrowth syndromes with complex vascular anomalies. Semin Pediatr Surg. 2014;23(4):208-15.

34. Clemens R, Pfammatter T, Meier T, Alomari A, Amann-Vesti B. Combined and complex vascular malformations. Vasa. 2015;44(2):92-105.
35. Martinez-Lopez A, Blasco-Morente G, Perez-Lopez I, Herrera-Garcia J, Luque-Valenzuela M, Sanchez-Cano D et al. CLOVES syndrome: review of a PIK3CA-related overgrowth spectrum (PROS). Clinical Genetics. 2016;91(1):14-21.

36. Lobo-Mueller E, Amaral JG, Babyn PS, Wang Q, John P. Complex combined vascular malformations and vascular malformation syndromes affecting the extremities in children. Semin Musculoskelet Radiol. 2009;13:255-76.

37. Alomari AI, Spencer SA, Arnold RW, Chaudry G, Kasser JR, Burrows PE et al. Fibro-adipose vascular anomaly: clinical-radiologic-pathologic features of a newly delineated disorder of the extremity. J Pediatr Orthop. 2014;34:109-17.

38. Kaduthodil MJ, Prasad DS, Lowe AS, Punekar AS, Yeung S, Kay CL. Imaging manifestations in Proteus syndrome- an unusual multisystem developmental disorder. Br J Radiol. 2012;85:e793-e9. 